\title{
Estudo cefalométrico individualizado do posicionamento da maxila em indivíduos com equilíbrio facial e oclusão normal*
}

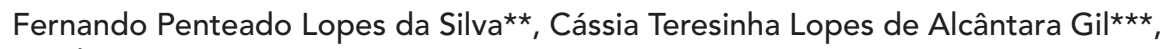

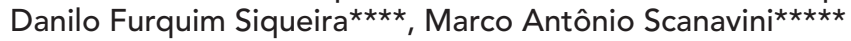

\section{Resumo}

Introdução: o posicionamento da maxila no esqueleto craniofacial tem sido motivo de investigação por diversos autores. Traduzindo suas idéias por meio de medidas lineares ou angulares, tais autores definiram o que consideraram como a posição "ideal", "normal" e, ainda, "aceitável" ou "inaceitável" da maxila, relacionando-a, na maioria das vezes, com a base do crânio. A partir da avaliação de indivíduos com oclusão considerada normal e com bom equilíbrio facial, eram calculados valores médios e desvios-padrão de determinadas medidas, os quais eram tomados como parâmetros para avaliações cefalométricas de pacientes distintos. Objetivos: diante das divergências de opiniões encontradas na literatura, a proposta do presente estudo foi avaliar o posicionamento da maxila nos sentidos vertical e ântero-posterior, além da sua inclinação, numa amostra de 94 indivíduos com oclusão normal e equilíbrio facial. Métodos: foram determinadas correlações entre medidas do próprio indivíduo: OPI-N com OPI-ENA e N-ENA com ENA-ENP. Conclusões: a partir dos fortes índices de correlação encontrados, concluiu-se que a medida OPI-N pode ser tomada como referência para determinação de OPI-ENA, da mesma forma que ENA-ENP pode ser considerada para determinação de N-ENA, definindo, respectivamente, a posição da maxila nos sentidos sagital e vertical. A inclinação da maxila, representada aqui pelo ângulo OPI.ENA.ENP, teve valor médio estatisticamente próximo a $0^{\circ}$ (zero grau), indicando forte tendência do prolongamento posterior do plano palatino (ENA-ENP) tangenciar a base posterior do crânio (ponto OPI), o que se revela como uma importante característica de indivíduos com oclusão normal e equilíbrio facial.

Palavras-chave: Individualização. Maxila. Oclusão normal. Cefalometria.

Artigo baseado na dissertação de mestrado do cirurgião-dentista Fernando Penteado Lopes da Silva, apresentada à Faculdade de Odontologia da Universidade Metodista de São Paulo - UMESP.

** Mestre em Ortodontia pela Universidade Metodista de São Paulo - UMESP.

*** Professora doutora do departamento de Ortodontia da Universidade Metodista de São Paulo - UMESP e orientadora da dissertação.

**** Professor doutor do departamento de Ortodontia da Universidade Metodista de São Paulo - UMESP.

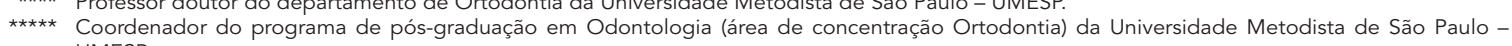
UMESP. 


\section{INTRODUÇÃO}

Sempre foi dada, por parte dos ortodontistas, atenção especial ao estudo da arquitetura craniofacial do "padrão normal", ou seja, da oclusão equilibrada associada a uma face harmoniosa.

Desde o início dos estudos em cefalometria, os autores buscam medidas angulares e lineares que traduzam o correto posicionamento das bases apicais entre si e destas com a base do crânio. Normalmente, por uma questão seqüencial na mensuração dos cefalogramas, os profissionais tendem, primeiramente, a examinar a relação da maxila com a base do crânio, para depois avaliar a condição da mandíbula.

O estudo cefalométrico, somado às análises facial e de modelos, é de grande importância na elaboração do diagnóstico ortodôntico. Os padrões lineares e angulares de cada análise cefalométrica, sejam relacionados aos dentes ou às bases apicais, são considerados, muitas vezes, como metas ao final do tratamento.

Muitos trabalhos foram realizados com intuito de buscar uma forma de análise cada vez mais adequada. Normalmente, são utilizadas medidas derivadas de grupos amostrais com oclusão considerada "normal" e com harmonia facial, na tentativa de obter valores aplicáveis a toda a população.

Porém, para autores como Sassouni ${ }^{21}$, Di Paolo et al. ${ }^{6}$ e Jefferson ${ }^{13}$, a maioria das análises não fornece informações confiáveis em relação à localização e à magnitude das desarmonias entre os maxilares, pois os pacientes avaliados têm que se enquadrar numa faixa de valores médios aceitáveis, com pouca ou nenhuma consideração ao seu padrão morfogenético individual.

As estruturas do esqueleto craniofacial, a exemplo de todo o corpo humano, são determinadas geneticamente, portanto normas cefalométricas tornam-se diferentes dependendo da raça, idade ou gênero do indivíduo. Dessa forma, não há uma regra geral que determine a posição ideal da maxila ou mandíbula no esqueleto craniofacial.
Desde estudos realizados na década de 50, como o de Riedel ${ }^{18}$, até os dias de hoje, com Jacobson e Sarver ${ }^{12}$, não há um consenso entre os diferentes autores e clínicos quanto ao posicionamento ideal que a maxila deve ocupar no esqueleto craniofacial.

A partir disso, enfocando o comportamento da maxila, a proposta do presente estudo é avaliar sua posição no esqueleto craniofacial de indivíduos com oclusão normal, de forma individualizada.

Para isso, foram feitas correlações entre medidas cefalométricas do próprio indivíduo, sem a comparação entre indivíduos diferentes. A partir dos resultados de tais correlações, foi verificada a possibilidade de aplicação das mesmas no diagnóstico e plano de tratamento de casos ortodônticos ou cirúrgicos.

\section{REVISÃO DA LITERATURA}

Angle², em 1899, afirmou que, apesar das faces humanas apresentarem semelhanças, todas elas são diferentes, impedindo que qualquer linha ou medida passa ter aplicação universal. Por essa razão, os ortodontistas deveriam ser capazes de detectar não apenas se o contorno da face do paciente está de acordo com um determinado padrão, mas também se os elementos faciais estão em equilíbrio e harmonia entre si. Para Angle, o melhor equilíbrio, a melhor harmonia e as melhores proporções das linhas faciais estariam associadas a uma oclusão com todos os dentes ocupando uma posição normal, sendo esta a única lei universal.

Coube a Downs ${ }^{7}$ o mérito da realização de um dos trabalhos pioneiros sobre valores normativos com validade estatística e comprovação clínica. Em sua pesquisa, o autor objetivou determinar a variação dos padrões facial e dentário em indivíduos com oclusão normal. Downs definiu um padrão facial médio representativo de indivíduos adolescentes jovens portadores de oclusão normal, concluindo também que há grande variação em torno desse padrão, a qual deve ser esperada quando se avalia o equilíbrio e a harmonia facial. 
Sassouni ${ }^{20}$ apresentou uma análise baseada na proporcionalidade entre quatro planos faciais horizontais, encontrada em indivíduos com oclusão normal e face equilibrada. $\mathrm{O}$ autor procurou estabelecer um padrão arquitetural básico, usando os seguintes planos de referência: (1) plano da base craniana ântero-superior; (2) plano palatino; (3) plano oclusal; e (4) plano da base mandibular. Em faces harmoniosas, esses quatro planos, prolongados posteriormente, encontram ou interceptam um ponto único, o Centro $\mathrm{O}$, localizado posteriormente ao contorno do osso occipital. Para avaliação da relação entre as bases apicais, traça-se o "arco anterior", com centro no Centro $\mathrm{O}$ e raio Centro O - N (Násio). Numa face equilibrada, o arco anterior deve tangenciar os pontos ENA e Pog. Nos casos com discrepância entre as bases apicais, o "arco anterior" é a referência para localização da causa da discrepância existente.

Em estudo posterior, Sassouni ${ }^{21}$ citou que o emprego de uma análise baseada na proporção geométrica da face do indivíduo parece mais racional e confiável, se comparada àquelas que utilizam valores numéricos semelhantes para avaliação de todos os casos. Além disso, esta abordagem torna mais fácil o entendimento da análise, pois evita comparações entre estruturas faciais e tabelas com valores numéricos. Para o autor, a correção de más oclusões baseada em normas cefalométricas absolutas pode se tornar um erro grave, sendo, muitas vezes, causa da recidiva dos tratamentos.

Para Di Paolo et al. ${ }^{6}$, a análise cefalométrica é uma importante ferramenta de diagnóstico para ortodontistas e cirurgiões. Porém, a maioria das análises utilizadas não fornece informações confiáveis em relação à localização e magnitude das desarmonias esqueléticas, já que os pacientes têm que se enquadrar numa faixa de valores médios aceitáveis, com pouca ou nenhuma consideração em relação ao padrão morfogenético individual. Segundo os autores, a grande combinação de variáveis do complexo craniofacial impossibilita que o profissional trate seus pacientes com objetivo de alcançar valores cefalométricos médios, havendo necessidade de uma abordagem individualizada.

$\mathrm{Gil}^{8}$, ao avaliar crânios de indivíduos adultos portadores de oclusão normal por meio de telerradiografias em norma lateral, frontal (PA) e axial, encontrou inúmeras medidas cefalométricas em proporção áurea. Segundo a autora, a avaliação das proporções áureas é uma forma de individualização do estudo cefalométrico, pois utiliza-se de medidas obtidas do próprio indivíduo, ao invés de medidas-padrão obtidas de médias amostrais.

No ano de 2001 em seu livro "Proporção áurea craniofacial", $\mathrm{Gil}^{9}$ destacou a coincidência entre o prolongamento do plano palatino (ENA-ENP) e o ponto Opístio (Op), localizado na região mais póstero-inferior da base do crânio, em indivíduos com oclusão normal, independente do padrão facial. Desta forma, a autora concluiu que a linha do plano palatino deveria ser considerada ao pensarse nas divisões naturais do crânio, sendo esta uma característica estrutural de indivíduos portadores de oclusão equilibrada.

Gil e Médici Filho ${ }^{10}$, estudando correlações existentes entre as proporções áureas em comum entre as linhas Op-N, Op-ENA e Op-Pog, encontraram igualdade significante entre estas medidas. Tais igualdades mostraram-se úteis à medida que, tomando-se como referência uma medida própria do paciente $(\mathrm{Op}-\mathrm{N})$, que não se altera com o tratamento, pode-se determinar o valor mais adequado de Op-ENA e Op-Pog, já que estas se apresentam iguais em indivíduos portadores de oclusão normal. Isso se torna possível, pois Op-ENA e Op-Pog são passíveis de abordagem terapêutica, como em casos ortodônticos ou orto-cirúrgicos.

Jacobson e Sarver ${ }^{12}$ relataram que a cirurgia ortognática para reposicionamento da maxila é um procedimento de grande importância na estética facial do paciente. Verificando a confiabilidade de métodos preditivos referentes a essas cirurgias, os autores encontraram coincidência da posição 
da maxila ao compararem os traçados preditivo e pós-operatório em $80 \%$ da amostra. Apesar da efetividade dos traçados avaliados, os autores concluíram que não há, ainda, um consenso sobre a posição ideal que a maxila deve ocupar no esqueleto craniofacial.

\section{MATERIAL E MÉTODOS}

A amostra avaliada foi constituída por 94 telerradiografias laterais de indivíduos brasileiros com dentadura permanente e oclusão clinicamente normal. Todos os indivíduos apresentaram equilíbrio entre os terços faciais, com selamento labial passivo. A média de idade foi de 19 anos (12 anos e 8 meses a 43 anos e 10 meses), sendo 41 pacientes do gênero masculino $(43,6 \%)$ e 53 do gênero feminino $(56,4 \%)$, sem tratamento ortodôntico prévio, sem extrações dentárias e com os segundos molares permanentes em oclusão.

Um dos critérios observados em todos os indivíduos foi a presença de, no mínimo, quatro das seis chaves de oclusão de Andrews ${ }^{1}$, sendo esse critério justificado pelo estudo de Maltagliati ${ }^{14}$. Em sua amostra de 97 brasileiros leucodermas com oclusão normal, a autora não encontrou nenhum caso que apresentasse as seis chaves de oclusão preconizadas por Andrews.

Todas as telerradiografias foram digitalizadas utilizando-se um scanner marca Hewlett Packard modelo 4C com leitor para transparências da mesma marca, modelo ScanJet 6100/CT. As imagens foram digitalizadas e importadas para o programa RadioCef Studio, específico para realização de traçados cefalométricos. A digitalização das imagens foi realizada com resolução de 150dpi no formato TIFF, sendo o dobro da resolução mínima exigida pelo programa.

Para realização das medições, foi necessária a criação do novo ponto cefalométrico OPI (Occipital Póstero-Inferior ${ }^{11}$, localizado no osso occipital. Todos os demais pontos utilizados faziam parte da lista de pontos anatômicos já existente no programa.
Desta forma, foram demarcados os seguintes pontos de referência (Fig. 1):

1. Occipital Póstero-Inferior (OPI): ponto mais póstero-inferior da base do crânio, localizado no osso occipital ${ }^{11}$.

2. Násio $(\mathrm{N})$ : ponto mais anterior da linha de união do osso frontal com os ossos próprios do nariz ${ }^{19}$.

3. Espinha Nasal Anterior (ENA): localizado na extremidade anterior da maxila ${ }^{19}$.

4. Espinha Nasal Posterior (ENP): localizado na parte mais posterior do palato ósseo ${ }^{19}$.

\section{Obtenção das grandezas cefalométricas}

Utilizando a ferramenta MixCef do programa RadioCef Studio (RadioMemory), criou-se a análise empregada no presente estudo, composta pelas seguintes medidas (Fig. 2 A, B):

1. OPI-N: distância entre os pontos OPI e N.

2. OPI-ENA: distância entre os pontos OPI e ENA.

3. N-ENA: distância entre os pontos $\mathrm{N}$ e ENA.

4. ENA-ENP: distância entre os pontos ENA e ENP.

5. OPI.ENA.ENP: ângulo formado pela li-

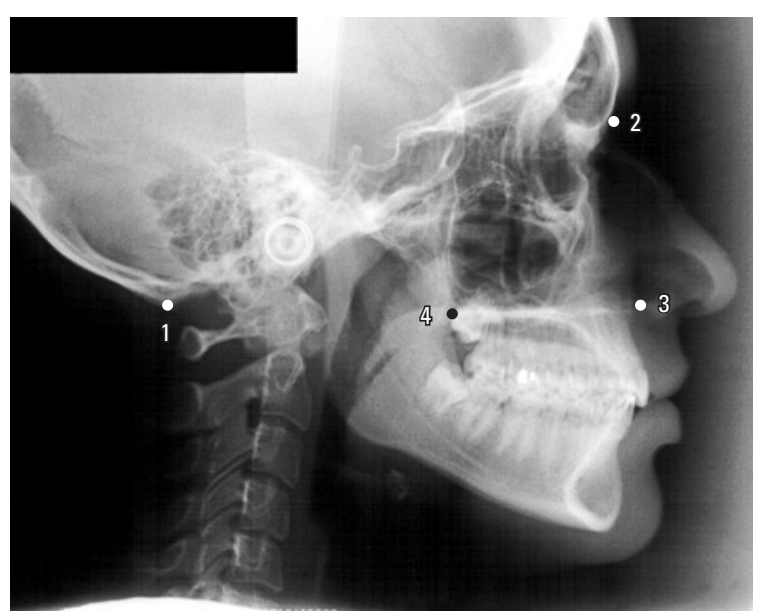

FIGURA 1 - Pontos cefalométricos utilizados: 1.0PI, 2.N, 3.ENA e 4.ENP. 

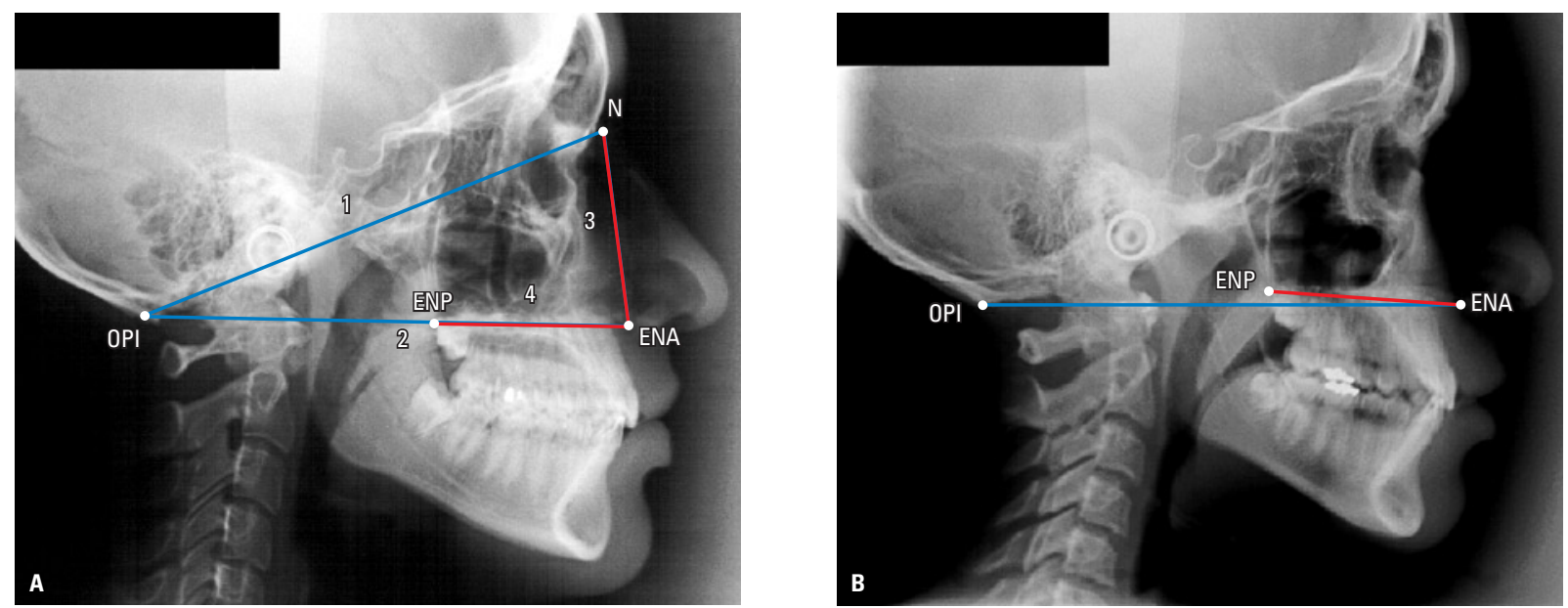

FIGURA 2 - A) Medidas lineares utilizadas: (1) OPI-N, (2) OPI-ENA, (3) N-ENA e (4) ENA-ENP. B) Medida angular OPI.ENA.ENP.

nha OPI-ENA e pelo plano palatino (ENA-ENP), tendo como vértice o ponto ENA.

Após a marcação dos pontos cefalométricos na telerradiografa digitalizada, o programa forneceu automaticamente o valor das medidas lineares e angulares.

Para avaliação do posicionamento da maxila no sentido ântero-posterior, foi adotada a correlação obtida entre as medidas OPI-N e OPI-ENA (Fig. 2A). A distância OPI-N, estável na faixa etária na qual se inclui a amostra e não passível de abordagem terapêutica, torna-se um referencial para determinação da distância OPI-ENA, definindo, assim, a posição sagital da maxila.

No sentido vertical, partindo do grau de correlação encontrado entre as medidas ENA-ENP e N-ENA (Fig. 2A), foi definida a possibilidade de utilizar-se a medida ENA-ENP para determinação do posicionamento vertical de ENA, a partir do ponto $\mathrm{N}$.

A avaliação da inclinação da maxila foi realizada por meio do ângulo OPI.ENA.ENP (Fig. 2B). $O$ referido ângulo é formado pelas linhas OPIENA e ENA-ENP, tendo como vértice o ponto ENA. Dessa forma, a inclinação do plano palatino (ENA-ENP) foi determinada em relação à base posterior do crânio, representada pelo ponto OPI.
Nos casos onde há coincidência entre as linhas OPI-ENA e ENA-ENP, o ângulo OPI.ENA.ENP tem valor $0^{\circ}$ (zero graus), indicando que o prolongamento do plano palatino tangencia a região póstero-inferior da base do crânio (ponto OPI). Já nos casos onde o plano palatino (ENA-ENP) está acima de OPI-ENA, o ângulo OPI.ENA.ENP tem valor positivo. Casos em que ENA-ENP está abaixo de OPI-ENA definem um valor negativo para OPI.ENA.ENP.

\section{Análise estatística}

Foram calculados média, desvio-padrão e valores máximo e mínimo para cada variável utilizada. Os testes estatísticos foram executados no programa Statistica for Windows v. 5.1 (StatSoft Inc., USA). Para a comparação entre as médias das medidas OPI-N com OPI-ENA e N-ENA com ENA-ENP foi utilizado o teste $t$ pareado. Para verificar a correlação entre as medidas OPI$\mathrm{N}$ com OPI-ENA e N-ENA com ENA-ENP foi utilizado o coeficiente de correlação de Pearson. Para comparação da medida OPI.ENA.ENP com a média esperada de zero, foi utilizado o teste $t$ de Student.

Em todos os testes estatísticos adotou-se um nível de significância de $5 \% 24$. 
Tabela 1 - Média, desvio-padrão e teste t pareado para comparação entre as medidas OPI-N e OPI-ENA.

\begin{tabular}{ccccccc}
\multicolumn{2}{c}{ OPI-N } & \multicolumn{2}{c}{ OPI-ENA } & diferença & $t$ & $p$ \\
média & d.p. & média & d.p. & & & \\
\hline 150,55 & 5,97 & 146,37 & 6,44 & 4,18 & 13,474 & $0,000^{*}$
\end{tabular}

* diferença estatisticamente significante $(p<0,05)$.

\section{RESULTADOS}

A comparação entre as médias das medidas OPI-N com OPI-ENA pelo teste t pareado é exibida na tabela 1 e o gráfico de correlação, assim como o coeficiente de correlação de Pearson para essas medidas, é mostrado no gráfico 1 .

Embora tenha havido diferença $(4,18 \mathrm{~mm})$ estatisticamente significante entre as médias das medidas OPI-N e OPI-ENA (Tab. 1), pode-se observar uma forte correlação $(r=0,89)$, estatisticamente significante (Gráf. 1).

Para detalhar as diferenças entre OPI-N e OPIENA, foi elaborado um histograma representativo dessa diferença para os 94 indivíduos da amostra (Gráf. 2).

A comparação entre as médias das medidas $\mathrm{N}$ ENA com ENA-ENP pelo teste $t$ pareado é mostrada na tabela 2 e o gráfico de correlação, assim como o coeficiente de correlação de Pearson, é mostrado no gráfico 3 .

A diferença entre as médias das medidas $\mathrm{N}$ ENA e ENA-ENP $(0,34 \mathrm{~mm})$ não foi estatisticamente significante (Tab. 2). A correlação observada foi forte, com tendência a muito forte $(\mathrm{r}=$ 0,87), e estatisticamente significante (Gráf. 3).

Para detalhar as diferenças entre N-ENA e ENA-ENP para os 94 indivíduos avaliados, foi elaborado o histograma do gráfico 4 .

Com relação à medida OPI.ENA.ENP, os dados da média, desvio-padrão, valor mínimo e valor máximo são apresentados na tabela 3 .

Casos onde houve sobreposição entre o plano palatino (ENA-ENP) e a linha OPI-ENA indicaram valor angular igual a $0^{\circ}$ (zero graus). As leituras angulares foram positivas quando ENA-ENP estava

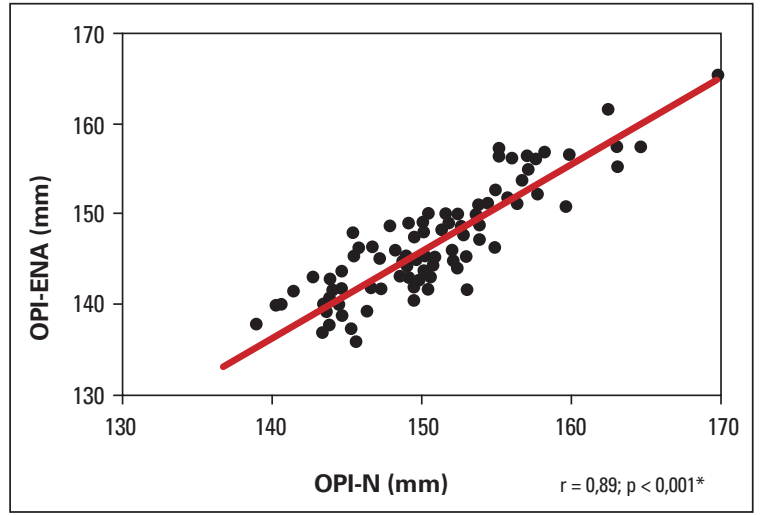

GRÁFICO 1 - Gráfico de dispersão e correlação de Pearson (r) entre as medidas OPI-N e OPI-ENA.

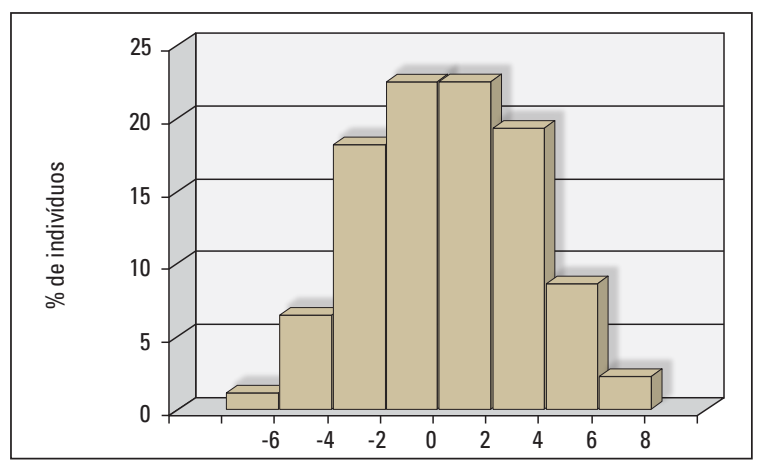

GRÁFICO 2 - Histograma da distribuição dos valores da diferença entre OPI-N e OPI-ENA para os 94 indivíduos da amostra.

acima de OPI-ENA e negativas quando ENA-ENP encontrava-se abaixo de OPI-ENA (Fig. 3).

Para comparação de OPI.ENA.ENP com a média esperada de $0^{\circ}$ (zero graus), aplicou-se o teste $t$ de Student e este não mostrou diferença estatisticamente significante $(\mathrm{t}=0,865 ; \mathrm{p}=0,389)$.

Os valores obtidos de OPI.ENA.ENP para os 94 sujeitos da amostra estão representados no histograma do gráfico 5 .

A distribuição gráfica dos valores de OPI.ENA. ENP encontrados na amostra está ilustrada no gráfico 6.

\section{DISCUSSÃO}

As medidas estudadas foram comparadas entre si para cada indivíduo, de forma a não se utilizar 
Tabela 2 - Média, desvio-padrão e teste t pareado para comparação entre as medidas N-ENA e ENA-ENP.

\begin{tabular}{ccccccc}
\multicolumn{2}{c}{ N-ENA } & \multicolumn{2}{c}{ ENA-ENP } & dife- & $t$ & $p$ \\
média & d.p. & média & d.p. & rença & & \\
53,66 & 3,19 & 53,32 & 3,35 & 0,34 & 1,955 & 0,054 n.s.
\end{tabular}

n.s. - diferença estatisticamente não significante.

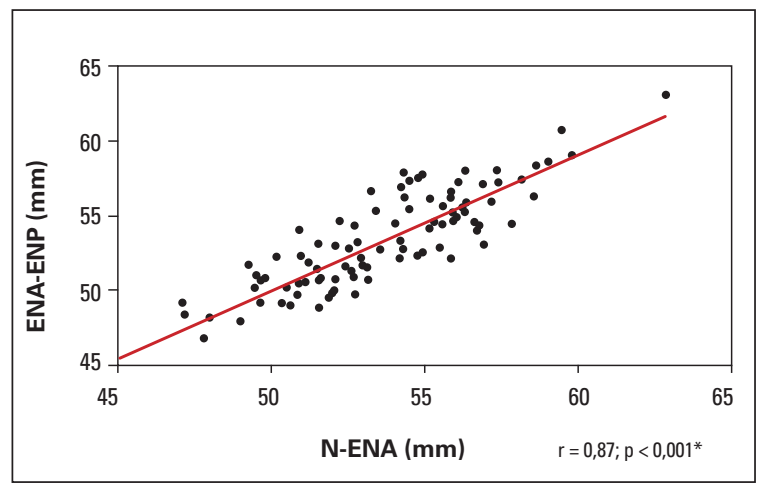

GRÁFICO 3 - Gráfico de dispersão e correlação de Pearson (r) entre as medidas N-ENA e ENA-ENP.
Tabela 3 - Média, desvio-padrão e valores mínimo e máximo para a medida OPI.ENA.ENP.

\begin{tabular}{ccccc}
\hline medida & média & d.p. & mínimo & máximo \\
$\begin{array}{c}\text { OPI.ENA.ENP } \\
\text { (graus) }\end{array}$ & $-0,15$ & 1,68 & $-4,09$ & 4,62 \\
\hline
\end{tabular}

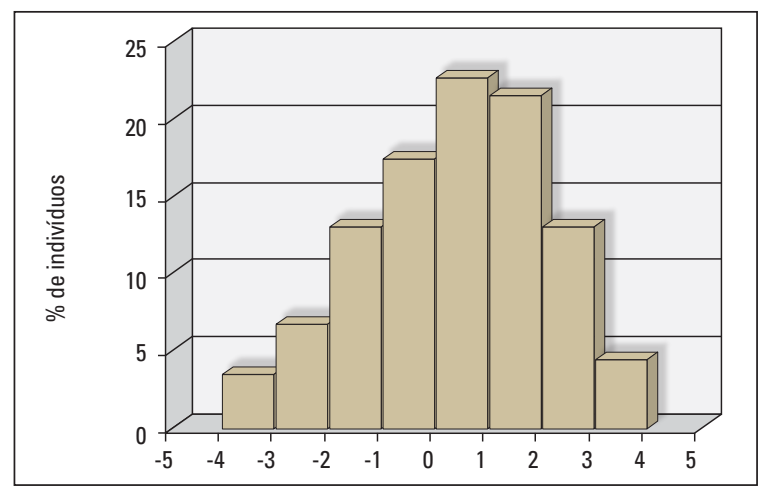

GRÁFICO 4 - Histograma da distribuição dos valores da diferença entre N-ENA e ENA-ENP para os 94 indivíduos da amostra.
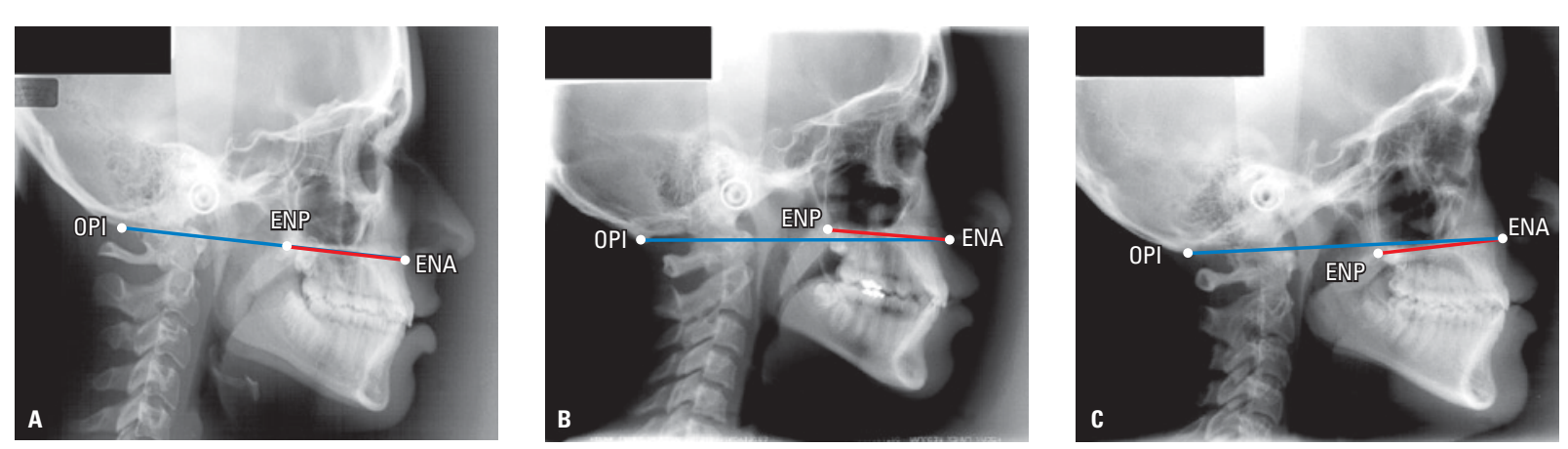

FIGURA 3 - A) Sobreposição de ENA-ENP e OPI-ENA (valor angular igual a zero). B) Plano ENA-ENP acima de OPI-ENA (valor angular positivo). C) Plano ENA-ENP abaixo de OPI-ENA (valor angular negativo).

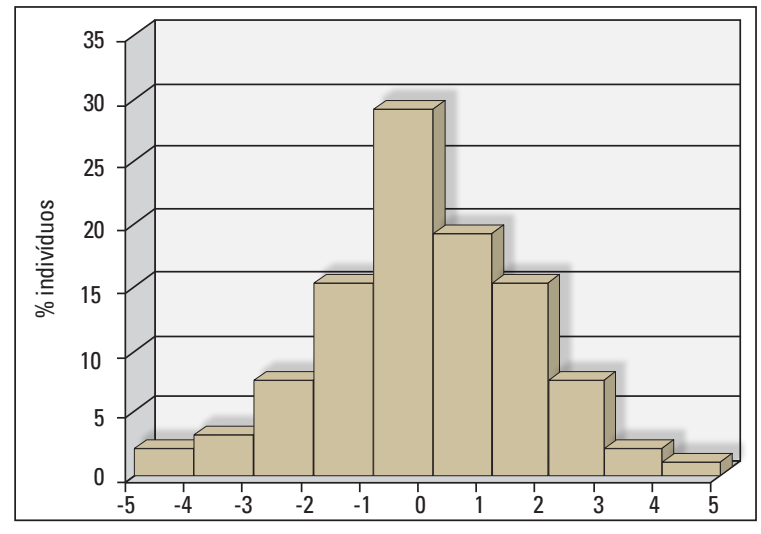

GRÁFICO 5 - Histograma da distribuição dos valores de OPI.ENA.ENP para os 94 indivíduos da amostra.

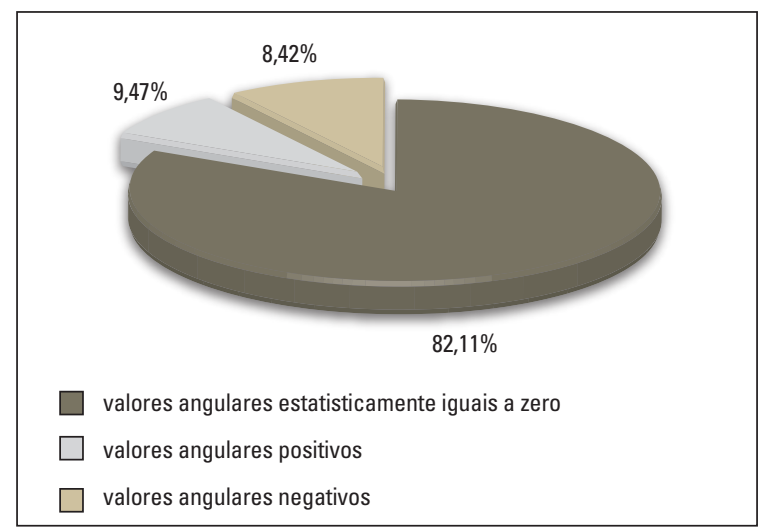

GRÁFICO 6 - Representação gráfica da distribuição dos indivíduos da amostra em relação aos valores de OPI.ENA.ENP. 
valores médios para representar toda a amostra. Com isso, as correlações obtidas entre as medidas avaliadas, demonstradas pelos testes estatísticos, caracterizaram a individualização da metodologia proposta.

Os 94 indivíduos avaliados tinham média de idade de 19 anos (12 anos e 8 meses a 43 anos e 10 meses), tendo já passado da época de crescimento significativo da maxila para ambos os gêneros, segundo Savara e Singh ${ }^{22}$. Ao avaliar o crescimento craniofacial em indivíduos dos 8 aos 22 anos de idade, Brodie ${ }^{4}$ constatou que, naqueles pertencentes à faixa etária semelhante à do presente estudo (média de 19 anos), ocorreu apenas um crescimento residual na região do ponto ENA em direção anterior, considerado inexpressivo.

A avaliação do posicionamento da maxila no presente trabalho se baseou em quatro medidas lineares, sendo utilizada apenas uma medida angular, para avaliação do ângulo do plano palatino em relação à base posterior do crânio. Miller et al. ${ }^{16}$; Baumrind, Frantz ${ }^{3}$ e Midtgard, Björk e Linder-Aronson ${ }^{15}$ constataram que grande parte dos erros de mensuração ocorre devido à incorreta marcação dos pontos cefalométricos. No presente estudo, foram utilizados quatro pontos, diminuindo as chances de erros inerentes às suas marcações. Os pontos utilizados foram o Occipital PósteroInferior (OPI) ${ }^{11}$, Násio $(\mathrm{N})^{19}$ e Espinha Nasal Anterior (ENA) ${ }^{19}$ e Posterior (ENP) ${ }^{19}$, os quais não apresentam divergências de conceituação quanto às suas localizações. Além disso, com exceção do ponto OPI, todos os outros são comumente utilizados em outras análises cefalométricas, sendo bem conhecidos pelos profissionais.

Gil et al. ${ }^{11}$, ao desenvolverem sua metodologia para localização do ponto OPI, verificaram pequena variação na marcação do referido ponto, tendo esta ocorrido apenas no sentido ântero-posterior, não havendo variação no sentido vertical. Variações na marcação de OPI em sentido horizontal, no caso do presente estudo, não interferem na leitura do ângulo OPI.ENA.ENP, pois tais varia- ções não provocam alteração na inclinação da linha OPI-ENA, constituinte do ângulo. Alterações das medidas lineares OPI-N e OPI-ENA, devido à variação na marcação de OPI, ocorreriam na mesma proporção, já que ambas partem desse mesmo ponto. Dessa forma, não haveria alteração no grau de correlação entre ambas as medidas, quando estas fossem comparadas entre si.

\section{Posição sagital da maxila (correlação entre OPI-N e OPI-ENA)}

Para cada indivíduo da amostra, foi aplicado o teste de correlação de Pearson para comparação entre essas duas medidas. Como resultado, obtevese um forte índice de correlação entre as mesmas $(r=0,89)$, tendendo a muito forte, sendo que uma correlação perfeita (igualdade absoluta) teria um fator $r=1,0$. A correlação entre OPI-ENA e OPI-N (Fig. 4) torna a avaliação de OPI-ENA individualizada, à medida que correlaciona duas medidas do próprio indivíduo, em vez de se estabelecer uma única média da distância OPI-ENA para toda a amostra.

A distância OPI-N, não passível de abordagem terapêutica, torna-se uma referência na determinação da distância OPI-ENA, pois ambas as me-

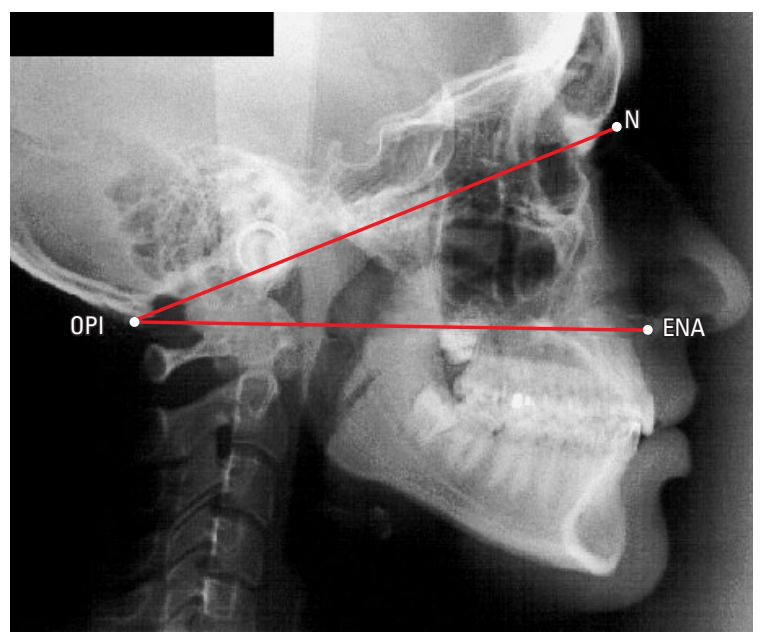

FIGURA 4 - Correlação entre OPI-N e OPI-ENA para avaliação sagital da maxila. 


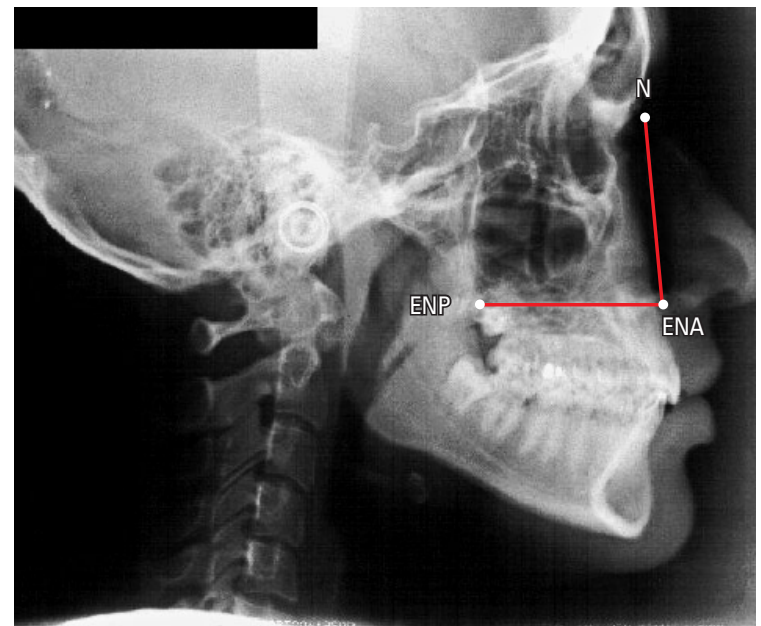

FIGURA 5 - Correlação entre N-ENA e ENA-ENP para avaliação vertical da maxila.

didas, considerando a amostra de oclusão normal avaliada, apresentaram forte correlação. A possibilidade de aplicação dessa correlação torna-se de grande auxílio para o diagnóstico e planejamento de casos onde a maxila apresentar-se comprometida no sentido ântero-posterior, já que, a partir do ponto OPI, pode-se estimar a posição ânteroposterior de ENA, pela equivalência entre as distâncias OPI-N e OPI-ENA.

\section{Posição vertical da maxila (correlação entre $\mathbf{N}$-ENA e ENA-ENP)}

Aplicando-se o teste de correlação de Pearson para avaliação de ambas as medidas, encontrouse o índice $r=0,87$, indicando correlação forte $\mathrm{e}$ estatisticamente significante. A principal aplicação desta correlação é a possibilidade de basear-se no comprimento do plano palatino (ENA-ENP) para se determinar o posicionamento do ponto ENA no sentido vertical, a partir do ponto $\mathrm{N}$.

Dessa forma, no plano sagital, aplicando-se o conceito de igualdade entre as medidas OPI-N e OPI-ENA, é possível determinar-se a posição do ponto ENA no sentido ântero-posterior. Para localizar o referido ponto no sentido vertical, a partir

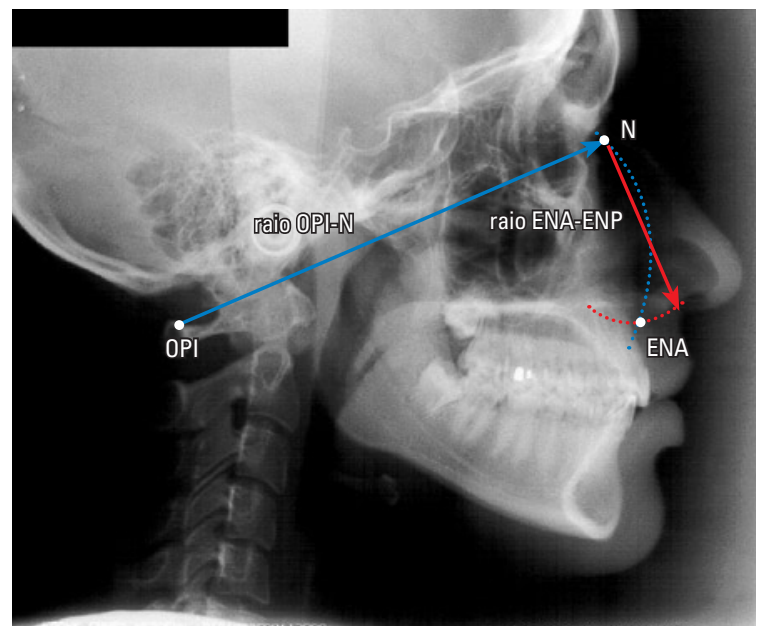

FIGURA 6 - Aplicação das correlações obtidas, em indivíduos com oclusão normal, para determinação do posicionamento da maxila: a partir de OPI, é estabelecido o limite anterior da maxila por meio da correlação entre OPI-N e OPI-ENA; a partir de N, é estabelecida a altura do ponto ENA, utilizando-se a correlação entre N-ENA e ENA-ENP.

do ponto $\mathrm{N}$, utiliza-se a igualdade entre ENA-ENP e N-ENA (Fig. 6).

Riolo et al. ${ }^{19}$ avaliaram longitudinalmente 83 indivíduos, 47 do gênero masculino e 36 do feminino, dos 6 aos 16 anos de idade, não-tratados ortodonticamente. Os dados obtidos pelos autores indicaram grande semelhança entre as medidas $\mathrm{N}$ ENA e ENA-ENP, característica que se manteve com o aumento da idade, dentro da faixa etária avaliada. Esses resultados reforçam a idéia de que o forte índice de correlação encontrado entre ambas as medidas no presente trabalho não é apenas característica de indivíduos adultos. Conseqüentemente, o emprego dessa correlação com finalidade de diagnóstico e planejamento não se limita apenas a indivíduos após a fase de crescimento. Pacientes jovens com diagnóstico de excesso vertical de maxila, por exemplo, podem ter como referência a distância ENA-ENP para quantificar esse excesso, bem como para avaliação dos resultados nos casos de emprego de terapia ortopédica.

\section{Avaliação da inclinação do plano palatino (ângulo OPI.ENA.ENP)}

No presente trabalho, a inclinação do plano 


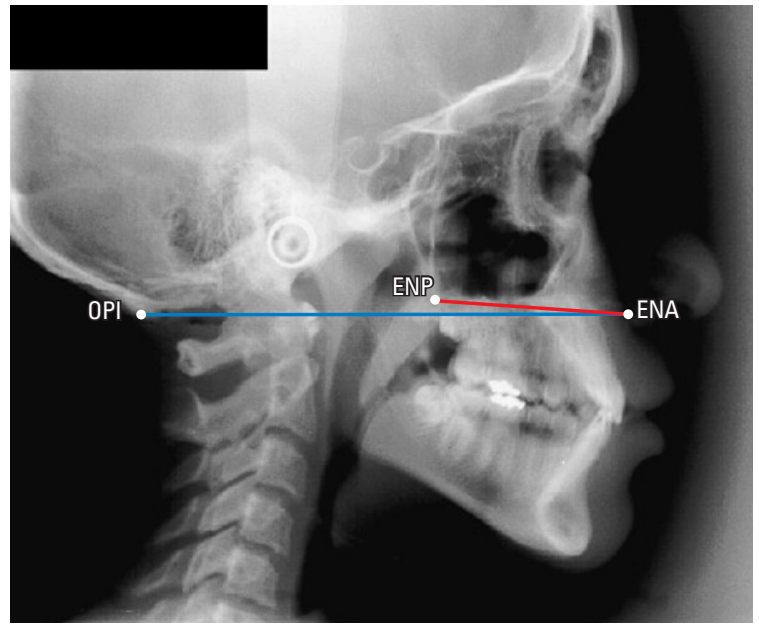

FIGURA 7 - Avaliação da inclinação do plano palatino: linhas OPI-ENA e ENAENP formando o ângulo OPI.ENA.ENP, tendo como vértice o ponto ENA.

palatino foi estudada por meio do ângulo formado pelas linhas OPI-ENA e ENA-ENP (Fig. 7), tendo como vértice o ponto ENA. Casos onde o ângulo OPI.ENA.ENP se aproximava do valor $0^{\circ}$ (zero graus) indicaram tendência à coincidência entre as linhas OPI-ENA e ENA-ENP, o que equivale a dizer que o prolongamento do plano palatino, nesses casos, tendeu a tangenciar a região póstero-inferior da base do crânio, ou seja, o ponto OPI.

A média encontrada para OPI.ENA.ENP foi de $-0,15^{\circ}$, com desvio-padrão de $\pm 1,68^{\circ}$. Aplicando-se o teste $t$ de Student para os valores angulares, verificou-se que os mesmos mostraramse estatisticamente muito próximos a $0^{\circ}$. Tais resultados indicaram forte tendência do prolongamento do plano palatino tangenciar a base posterior do crânio.

$\mathrm{Gil}^{9}$, no ano de 2001, em seu livro "Proporção áurea craniofacial", destacou a coincidência entre o prolongamento do plano palatino (ENA-ENP) e o ponto Op (Opístio), localizado na parte posterior da base do crânio. Essa característica foi encontrada em toda a amostra com oclusão normal estudada, independente do padrão facial dos indivíduos. Desta forma, a autora concluiu que a linha do plano palatino deveria ser considerada ao pensar-se nas divisões naturais do crânio, sendo esta uma característica da oclusão normal.

No presente estudo, o procedimento de medição do ângulo do plano palatino, tendo como referência a base posterior do crânio (ponto OPI), mostrou-se de fácil execução. A média encontrada $\left(-0,15 \pm 1,68^{\circ}\right)$ indicou um pequeno desvio-padrão, se comparado ao de outros trabalhos envolvendo amostras de indivíduos com oclusão normal, como Cangialosi ${ }^{5}$, que encontrou uma média de $7,1 \pm 3,7^{\circ}$ para o ângulo PP.S-N.

O desvio-padrão de OPI.ENA.ENP $\left( \pm 1,68^{\circ}\right)$ indica ser esta uma medida que varia menos de um indivíduo para outro, em relação a outras medidas como, por exemplo, PP.PHF, a qual mostrou desvio-padrão de $\pm 3,96^{\circ}$ na amostra de $\mathrm{Urbano}^{23}$, e de $\pm 3,5^{\circ}$ na amostra de Ricketts ${ }^{17}$. A homogeneidade da amostra com relação ao ângulo OPI. ENA.ENP também pode ser constatada pela distribuição equilibrada entre os valores positivos e negativos do referido ângulo (Gráf. 7).

\section{CONSIDERAÇÕES FINAIS}

A partir do emprego das correlações aqui obtidas, pode-se visualizar a posição considerada "normal" da maxila, elaborando, assim, uma predição da posição da mesma, baseando-se nos achados da oclusão normal do presente estudo.

Contudo, há necessidade de estudos com apli-

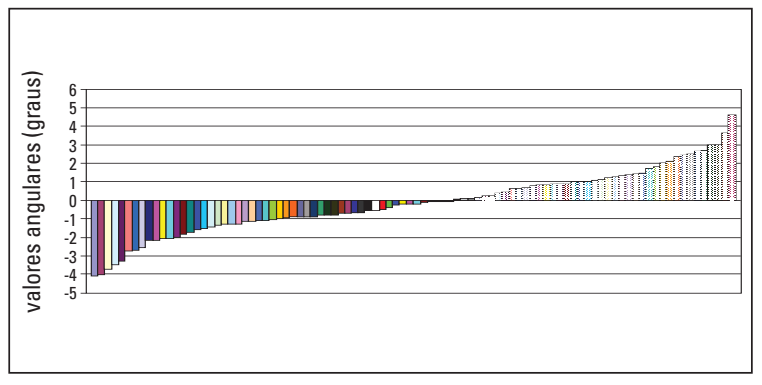

GRÁFICO 7 - Distribuição dos valores angulares de OPI.ENA.ENP na amostra estudada. 
cação clínica para que as correlações encontradas tenham seus resultados terapêuticos avaliados. A partir do presente trabalho, serão necessários estudos direcionados ao emprego de tais correlações no diagnóstico e planejamento de casos com comprometimento maxilar.

Considerando o termo individualização como um sinônimo de respeito aos pacientes, este estudo pretende auxiliar no diagnóstico e planejamento ortopédico ou ortodôntico-cirúrgico do posicionamento da maxila, contribuindo para o restabelecimento da harmonia facial.

\section{CONCLUSÃO}

Baseado na análise e discussão dos resultados da amostra de oclusão normal avaliada e de acordo com a metodologia empregada, conclui-se que:

1) A distância OPI-N mostrou ser uma referência aplicável para avaliação da posição sagital da maxila, pois a partir dela pode-se determinar a distância OPI-ENA.
2) A distância ENA-ENP mostrou ser uma referência aplicável para avaliação da posição vertical da maxila, pois a partir do ponto $\mathrm{N}$ pode-se determinar a distância N-ENA, baseando-se em sua forte correlação com ENA-ENP.

3) Existe forte tendência do prolongamento posterior do plano palatino tangenciar a região póstero-inferior do crânio, representada pelo ponto OPI. Dessa forma, em conjunto com as correlações previamente encontradas, a inclinação do plano palatino completa a avaliação do posicionamento da maxila, pois fornece uma referência para o posicionamento vertical da Espinha Nasal Posterior (ENP).

\title{
Individual cephalometric study of the position of the maxilla in subjects with good facial balance and normal occlusion
}

\begin{abstract}
Introduction: The position of the maxilla in the skull is subject of investigation by many authors. Transmitting their ideas trough linear and angular measurements, they defined what is considered as "ideal", "normal" and "acceptable" or "unacceptable" position of the maxilla, relating it in most of the time with the cranial base. By the study of subjects with considered "normal" occlusion and good facial balance, it was calculated mean values and standard deviations of some measurements, which were considered as parameters to evaluate all kinds of malocclusions. Aim: Considering the divergences found in the literature, the aim of the present study was to evaluate the vertical and horizontal position of the maxilla and its inclination, in 94 subjects with normal occlusion and good facial balance. Methods: Considering this, it was determined some correlations between measurements of the own subjects: OPI-N with OPI-ENA and N-ENA with ENA-ENP. Conclusions: By the strong index of correlation found between these measurements, it was concluded that OPI-N can be take as a reference to establishment of OPIENA. In addition, ENA-ENP can be considered a reference to the distance N-ENA, so it is possible to determine respectively the position of the maxilla on the horizontal and vertical planes. The inclination of the maxilla, defined by the angle OPI.ENA.ENP, showed a mean value statistically very close of $0^{\circ}$ (zero), indicating a strong tendency of the posterior extension of the palatal plane (ENA-ENP) to touch the most posterior and inferior region of the occipital bone (OPI point). This was considered an important characteristic of subjects with normal occlusion and good facial balance.
\end{abstract}

Key words: Individualization. Maxilla. Normal occlusion. Cephalometrics. 


\section{REFERÊNCIAS}

1. ANDREWS, L. F. The six keys to normal occlusion. Am. J. Orthod., St. Louis, v. 62, no. 3, p. 296-309, Sept. 1972.

2. ANGLE, E. H. Classification of malocclusion. Dental Cosmos, Philadelphia, v. 41, no. 3, p. 248-264, 1899.

3. BAUMRIND, S.; FRANTZ, R. C. The reliability of head measurements. Am. J. Orthod., St. Louis, v. 60, no. 5, p. 505-517, Nov. 1971.

4. BRODIE, A. G. Late growth changes in the human face. Angle Orthod., Appleton, v. 23, no. 3, p. 146-157, July 1953.

5. CANGIALOSI, T. J. Skeletal morphologic features of anterior open bite. Am. J. Orthod., St. Louis, v. 85, no. 1, p. 28-36, Jan. 1984.

6. DI PAOLO, R. J. et al. The quadrilateral analysis: an individualized skeletal assessment. Am. J. Orthod., St. Louis, v. 83 , no. 1, p. 19-32, Jan. 1983

7. DOWNS, W. B. Variations in facial relationships: their significance in treatment and prognosis. Am. J. Orthod., St. Louis, v. 34, p. 812-840, 1948

8. GIL, C. T. L. A. Estudo da proporção áurea na arquitetura do crânio de indivíduos com oclusão normal, a partir de telerradiografias laterais, frontais e axiais. 1999. $183 \mathrm{f}$. Tese (Doutorado) - Faculdade de Odontologia, Campus de São José dos Campos, Universidade Estadual Paulista "Júlio de Mesquita Filho",1999.

9. GIL, C. T. L. A. Proporção áurea craniofacial. 1. ed. São Paulo: Ed. Santos, 2001.

10. GIL, C. T. L. A.; MÉDICI FILHO, E. Estudo da proporção áurea na arquitetura craniofacial de indivíduos com oclusão normal, a partir de telerradiografias axiais, frontais e laterais. Ortodontia, São Paulo, v. 35, n. 2, p. 69-85, abr./jun. 2002

11. GIL, C. T. L. A. et al. Determinação e localização do ponto OPI em telerradiografias em norma lateral. Rev. Odontol. UMESP São Paulo, ano 12, n. 23, p. 63-73, jan./jun. 2004.

12. JACOBSON, R.; SARVER, D. M. The predictability of maxillary repositioning in Le Fort I orthognathic surgery. Am. J. Orthod. Dentofacial Orthop., St. Louis, v. 122, no. 2, p. 142-154, Aug. 2002.

13. JEFFERSON, Y. Skeletal types: key to unraveling the mystery of facial beauty and its biologic significance. J. Gen. Orthod. Milwaukee, v. 7, p. 7-25, June 1996
14. MALTAGLIATI, L. A. B. Prevalência das seis chaves de oclusão de Andrews em jovens brasileiros com oclusão normal. Rev. Assoc. Paul. Cir. Dent., São Paulo, v. 55, n. 6, p. 411-415, nov./dez. 2001

15. MIDTGARD, J.: BJÖRK, G.; LINDER-ARONSON, S

Reproducibility of cephalometric landmarks and errors of measurements of cephalometric cranial distances. Angle Orthod., Appleton, v. 44, p. 56-61, 1974

16. MILLER, P. A. et al. Analysis of errors in cephalometric measurements of three-dimensional distances on the maxilla. Angle Orthod., Appleton, v. 36, no. 2, p. 169-175, Apr. 1966.

17. RICKETTS, R. M.; A foundation for cephalometric communication. Am. J. Orthod., St. Louis, v. 44, no. 5, p. 330-357, May 1960.

18. RIEDEL, R. A. The relation of maxillary structures to cranium in malocclusion and in normal occlusion. Angle Orthod. Appleton, v. 22, no. 3, p. 142-145, 1952.

19. RIOLO, M. L. et al. An atlas of craniofacial growth: cephalometric standards from the University School Growth Study. Michigan: The University of Michigan, 1974. Monograph no. 2, Craniofacial Growth Series, 379. p. 1974.

20. SASSOUNI, V. A. A roentgenographic cephalometric analysis of cephalo-facio-dental relationships. Am. J. Orthod., St. Louis v. 41, no. 10, p. 735-764, Oct. 1955.

21. SASSOUNI, V. A. Diagnosis and treatment planning via roentgenographic cephalometry. Am. J. Orthod., St. Louis, v. 44, no. 6, p. 433-463, June 1958

22. SAVARA, B. S.; SINGH, I. J. Norms of size and annual increments of seven anatomical measures of maxillae in boys from three to sixteen years of age. Angle Orthod., Appleton, v. 38 , no. 2, p. 104-120, Apr. 1968

23. URBANO, A. L. Avaliação cefalométrica radiográfica de jovens leucodermas com oclusão normal, segundo a análise lateral de Ricketts. 2003. 195 f. Dissertação (Mestrado) Faculdade de Odontologia, Universidade Metodista de São Paulo, São Bernardo do Campo, 2003

24. ZAR, J. H. Bioestatistical analysis. 3rd ed. New Jersey Prentice-Hall, 1996.
Endereço para correspondência

Fernando Penteado Lopes da Silva

Rua Sampaio Ferraz, 570 - Cambuí

CEP: 13.024-431 - Campinas / SP

E-mail: penteado_fer@yahoo.com.br 